\title{
Decreased serum levels of IL-27and IL-35 in patients with Graves disease
}

\author{
Malek-Hosseini Saeed ${ }^{1}$ \\ https://orcid.org/0000-0002-5883-6922 \\ Kalantar Kurosh ${ }^{1}$ \\ https://orcid.org/0000-0002-9160-9449
}

Amirghofran Zahra ${ }^{1,2}$

https://orcid.org/0000-0002-1027-7906

Dabbaghmanesh Mohamad Hossein ${ }^{3}$

https://orcid.org/0000-0002-4877-0376

Rostamzadeh Davood ${ }^{1}$

https://orcid.org/0000-0002-5528-1984

Mohammad Reza Ataollahi ${ }^{4}$

https://orcid.org/0000-0001-8007-4736

\author{
1 Department of Immunology, \\ School of Medicine, Shiraz \\ University of Medical \\ Sciences, Shiraz, Iran \\ ${ }^{2}$ Autoimmune Diseases Research \\ Center, Shiraz University of \\ Medical Sciences, Shiraz, Iran \\ ${ }^{3}$ Endocrine and Metabolism \\ Research Center, Nemazee \\ Hospital, Shiraz University of \\ Medical Sciences, Shiraz, Iran \\ ${ }^{4}$ Department of Immunology, \\ School of Medicine, Fasa University \\ of Medical Sciences, Fasa, Iran
}

\begin{abstract}
Objectives: Graves' disease (GD) is an autoimmune disease causing the overproduction of the thyroid hormone from thyroid gland. This disease is mainly the result of the production of antibodies against TSH receptors. Cytokines play an important role in orchestrating the pathophysiology in autoimmune thyroid disease. The regulatory role of IL-12 on TH1 cells has been proven. IL-27 and IL-35, members of IL-12 cytokine family, are two cytokines that have been newly discovered. IL-35 has been identified as a novel immunosuppressive and anti-inflammatory cytokine while IL-27 has both inflammatory and anti-inflammatory functions. The objective of the current study was to examine the changes in the serum level of the foregoing cytokines in GD patients in comparison to healthy controls. Materials and methods: In this study, serum levels of IL-27 and IL-35 were determined by an ELISA method; anti TPO and anti Tg were measured by an RIA method in 40 new cases of Graves's disease. The findings were compared with 40 healthy controls. Results: The results showed a significant difference between IL-27 and IL-35 regarding their serum levels with P values of 0.0001 and 0.024 , respectively; anti TPO and anti Tg levels of the cases were also significantly different from controls $(p<0.001)$. Conclusion: The reduction in the serum levels of IL-27 and IL-35 in GD patients compared to normal subjects suggests the possible anti-inflammatory role of these cytokines in GD. Arch Endocrinol Metab. 2020;64(5):521-7
\end{abstract}

Keywords

Graves' disease; IL-27; IL-35; anti-TP0; anti-Tg
Correspondence to:

Mohammad Reza Ataollahi

Immunology Department,

School of Medicine,

Fasa University of Medical Sciences

Fasa, Iran

ataollahimr@gmail.com

Received on Mar/7/2019

Accepted on July/31/2019

DOI: 10.20945/2359-3997000000227

\section{INTRODUCTION}

G rave's disease (GD) is an autoimmune thyroid Idisease characterized by thyrotoxicosis, diffuse goiter and the presence of autoantibody against thyroidstimulating hormone (TSH) receptor (1). The three major clinical signs of this disease are hyperthyroidism, ophthalmopathy and localized skin manifestations (2). GD is reported in $0.5 \%$ of the world population and its incidence is 5-10 times higher in women than in men (3). Although GD is a multifactorial disease whose etiology has not been fully fathomed, evidence shows that the imbalance between pro- and anti-inflammatory cytokines and production of aberrant autoantibodies play a pivotal role in the disease pathogenesis (1). Many studies have shown that pro-inflammatory cytokines such as interleukin (IL)-2, IL-8, IL-6, tumor necrosis factor
(TNF)- $\alpha$ and IL-17 are increased in the serum of GD patients (4). The evaluation of the T helper (TH) subsets involvement in GD showed a TH2 bias immunological imbalance and an increase in THI- and THI7-related cytokines compared to healthy controls (5-7).

Cytokines play a crucial role in triggering and coordinating inflammatory immune responses. Improper cytokine expression appears to influence the pathogenesis of many human diseases including thyroid autoimmune diseases. The immune regulation of two cytokines related to IL-12 family, namely IL-27 and IL-35, has been demonstrated in several autoimmune diseases (8). IL-27 is a heterodimeric cytokine comprised of Epstein-Barr virus (EBV)induced gene 3 (EBI3) subunit (a protein linked to IL12-p40) and the p28 subunit (9). Antigen presenting 
cells, including monocytes, macrophages and dendritic cells are the most important sources of IL-27 (10) that has both pro-inflammatory and anti-inflammatory activities (11). This cytokine is able to induce the proliferation of naive $\mathrm{T}$ cells and promote $\mathrm{TH} \mathrm{l}$ immune responses (12). Additionally, IL-27 regulates the production of anti-inflammatory cytokines such as IL4 , IL-10, and transforming growth factor beta (TGF- $\beta$ ) (13). IL-35 is a novel cytokine also belonging to IL-12 family. It is produced by regulatory $\mathrm{T}$ cells and plays an important role in suppressing the immune system (14). Regulation of cytokine network is a main target of various investigations in autoimmune inflammatory diseases $(15,16)$. The immunoregulatory role of these two cytokines in GD is yet to be elucidated. Therefore, the aim of the present study was to generate a new insight on the role of these cytokines in GD development or pathogenesis. For this purpose, the levels of IL-27 and IL-35 cytokines were compared between GD patients and healthy controls. The possible relationship between these cytokines and the presence of anti-thyroid antibodies including anti-thyroid peroxidase (antiTPO) and anti-thyroglobulin (anti-Tg) antibodies was further assessed in GD patients.

\section{MATERIALS AND METHODS}

\section{Study population}

Forty new cases of GD referring to the Motahari outpatient clinics, Shiraz University of Medical Sciences, participated in this study. Patients were diagnosed by an endocrinologist on the basis of clinical manifestations, biochemical criteria of thyrotoxicosis ( TSH $<0.05 \mathrm{mIU} / \mathrm{L}$ and increased free T3 and/or free T4 levels) and the presence of TSH receptor antibodies (TRAb). The Graves orbitopathy (GO) was checked in GD patients according to the NO SPECS grading system. Moreover, 40 healthy subjects matched in age and sex with the patient group were included in the study. The controls did not have any history of GD or other autoimmune diseases. Written informed consent was obtained from all the subjects, and the study was reviewed and approved by the Ethics Committee of Fasa University of Medical Sciences.

\section{Sample collection}

From all study participants, $5 \mathrm{ml}$ peripheral blood sample was collected. Following centrifugation at $3000 \mathrm{rpm}$ for $10 \mathrm{~min}$, the sera were separated, aliquoted and kept at $-70{ }^{\circ} \mathrm{C}$ until further use.

\section{Measurement of anti-Tg and anti-TPO and TSH receptor antibodies}

Serum levels of anti-Tg and anti-TPO antibodies were evaluated by radioimmunoassay (RIA) using anti-hTg $\left[{ }^{125} \mathrm{I}\right]$ RIA kit (RK-8CT) and anti-hTPO [ $\left.{ }^{125} \mathrm{I}\right]$ RIA kit (RK-36CT) obtained from Institute of ISOTOPE, Hungary. RIA protocol was carried out according to the manufacturer's instruction. Briefly, samples and calibrators were incubated together with biotin labelled anti-Tg (or biotin labelled anti-TPO for anti-TPO assay) and ${ }^{125} \mathrm{ITg}$ (or ${ }^{125} \mathrm{I}$ TPO) in the streptavidincoated tubes. Following incubation, the content of the tubes was aspirated and the bound activity was measured in a gamma counter. The concentrations of anti-Tg and anti-TPO antibodies were inversely proportional to the radioactivity measured in the test tubes. The concentration was read off the calibration curve generated through plotting the binding values against a series of calibrators containing known amounts of anti-Tg or anti-TPO.

Serum levels of TSH receptor antibody (TRAb) were evaluated by competitive ELISA technique (Medizym $^{\circledR}$ TRAb clone from Medipan, Dahlewitz/ Berlin, Germany) according to the manufacturer's instruction. Briefly, samples, control and calibrators were added to respective tubes and incubated for 120 min at room temperature. After incubation, wells were aspirated and washed to remove any residual droplets. Next, monoclonal human antibody biotin complex (M22) was added. The incubation and washing steps was repeated. Conjugate solution was then added to the test tubes. After that, the incubation and washing steps were performed, and the substrate solutions were then added to each well. Finally, the optical density was read at $450 \mathrm{~nm}$. The concentrations of TRAb were inversely proportional to the enzymatic activity measured in the test tubes.

\section{Measurement of serum IL-27 and IL-35 levels}

IL-27 and IL-35 cytokine serum levels were measured using ELISA kits from R\&D Systems, USA (for IL-27) and Zell Bio, Germany (for IL-35) according to the manufacturer's instructions. The sensitivity of tests was $12.8 \mathrm{pg} / \mathrm{mL}$ (IL-27) and $0.015 \mathrm{ng} / \mathrm{mL}$ (IL-35). Briefly, for IL-27 measurement, $100 \mu \mathrm{L}$ of capture antibody was 
coated in each well of a 96-well microplate overnight at room temperature (RT). After washing and blocking, $100 \mu \mathrm{L}$ serum samples of patients and controls were added for $2 \mathrm{~h}$ at RT. Plates were washed again and $100 \mu \mathrm{L}$ of the detection antibody and then $100 \mu \mathrm{L}$ of the working dilution of streptavidin-horseradish peroxidase (HRP) were added for $20 \mathrm{~min}$. The substrate solution $(100 \mu \mathrm{L})$ was then added for $20 \mathrm{~min}$, and finally the reaction was stopped by adding a stop solution to each well. Regarding IL-35 serum levels, $40 \mu \mathrm{L}$ samples along with $10 \mu \mathrm{L}$ anti-IL-35 biotinylated antibody and $50 \mu \mathrm{L}$ streptavidin-HRP were simultaneously added to a 96-well microplate pre-coated with anti-IL-35 antibody. After $60 \mathrm{~min}$ at $37^{\circ} \mathrm{C}$ and then washing, $100 \mu \mathrm{L}$ of substrate solution was added for $10 \mathrm{~min}$ at $37^{\circ} \mathrm{C}$, and the reaction was then stopped via a stopping solution. Ultimately, for both cytokines, the optical density of samples was read by use of a microplate reader at $450 \mathrm{~nm}$. The levels of cytokines were extrapolated from the related standard curve.

\section{Statistical analysis}

Statistical analysis and plotting of the graphs were performed using SPSS version 23 (SPSS Inc. Chicago, USA) and GraphPad Prism (GraphPad software Inc. CA) software, respectively. The non-parametric Mann-Whitney U-test was used to compare the serum levels of cytokines between patients and controls.
The correlation between the serum levels of IL-27 and IL-35 and autoantibodies was examined using the Pearson test. P-values less than 0.05 were considered significant.

\section{RESULTS}

\section{Patient's demographics}

In this study, 31 patients $(77.5 \%)$ were females and 9 were males. The mean age of patients was 35.9 years (range: $20-60$ years). Of the 40 healthy controls, 30 $(75 \%)$ were females and $10(25 \%)$ were males. The mean age and age range of controls were 36.5 and 2060 years, respectively.

\section{Detection of anti-TPO and anti-Tg antibodies and thyroid hormones}

In GD patients, the levels of free T4, free T3, TSH and TRAb were $32.1 \pm 9.30 \mathrm{pmol} / \mathrm{l}, 12.5 \pm 6.23$ $\mathrm{pmol} / 1,0.1 \pm 0.9 \mathrm{mIU} / \mathrm{mL}$ and $5.1 \pm 0.2 \mathrm{mIU} / \mathrm{mL}$, respectively. Twenty-two patients $(55 \%)$ were positive for anti-TPO antibody and 33 (82.5\%) were positive for anti- $\mathrm{Tg}$ antibody. All normal subjects were negative for these two antibodies. All GD patients were positive for TRAb (Table 1). Higher levels of anti-TPO and anti- $\mathrm{Tg}$ antibodies were observed in patients compared to the controls $(\mathrm{p}<0.001$, Figure 1$)$.

Table 1. Anti-Tg and anti-TPO antibody levels in patients and healthy controls

\begin{tabular}{|c|c|c|c|c|c|}
\hline & \multirow[b]{2}{*}{ Number } & \multicolumn{2}{|c|}{ Anti-TPO (IU/mL) } & \multicolumn{2}{|c|}{ Anti-Tg (IU/mL) } \\
\hline & & Range & Mean \pm SE & Range & Mean \pm SE \\
\hline Grave's patients & 40 & $12.1-696$ & $135 \pm 30.5$ & $39.9-1177$ & $299 \pm 42.4$ \\
\hline Control group & 40 & $7.3-59.1$ & $22.4 \pm 1.01$ & $31.1-223$ & $114.4 \pm 12.2$ \\
\hline
\end{tabular}
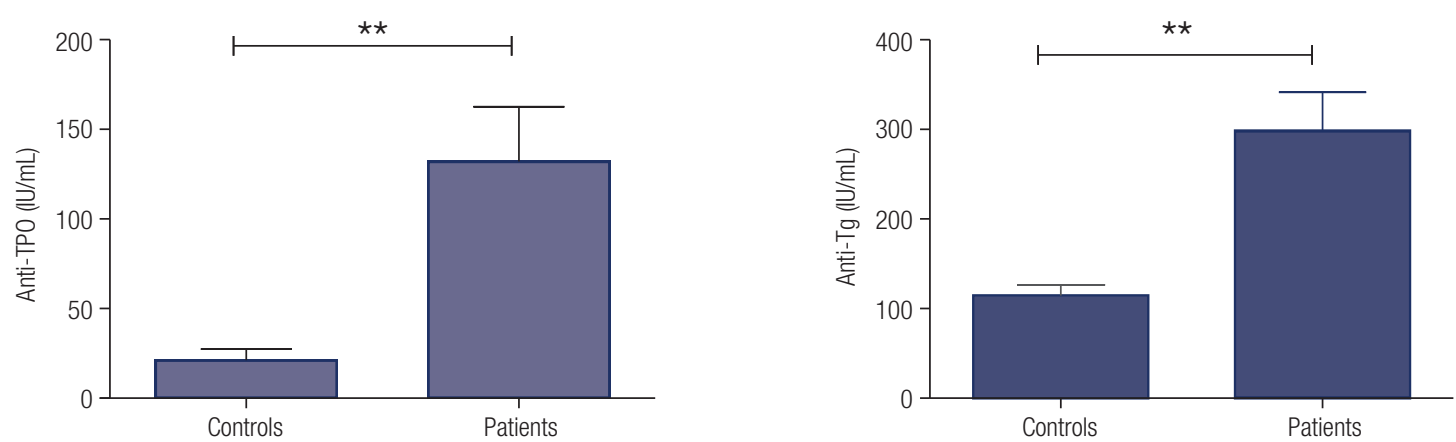

Figure 1. Anti-TPO and Anti-Tg levels in GD patients and healthy group. Serum antibody levels in GD patients $(n=40)$ and control group $(n=40)$ were measured by radioimmunoassay. Anti-TPO and anti-Tg levels were significantly higher in GD patients $\left({ }^{\star \star} p<0.001\right)$. 


\section{Serum levels of IL-27 and IL-35}

Analysis of the IL-27 cytokine level in the studied subjects showed a lower IL-27 serum concentration in the patients compared with the control group (3536 \pm 246.3 versus $6013 \pm 314.3 \mathrm{pg} / \mathrm{mL}$ ). As shown in Figure $2 \mathrm{~A}$, the difference between these levels was significant $(\mathrm{p}=0.024)$. Similarly, a lower IL-35 level was observed in the patients $(3.02 \pm 0.25 \mathrm{ng} / \mathrm{mL})$ in comparison to the controls $(7.12 \pm 0.36 \mathrm{ng} / \mathrm{mL})$, $(\mathrm{p}<0.0001)$ (Figure 2B). Statistical analysis showed no significant correlation between the IL-27 and IL-35 cytokines neither in patients nor in controls.

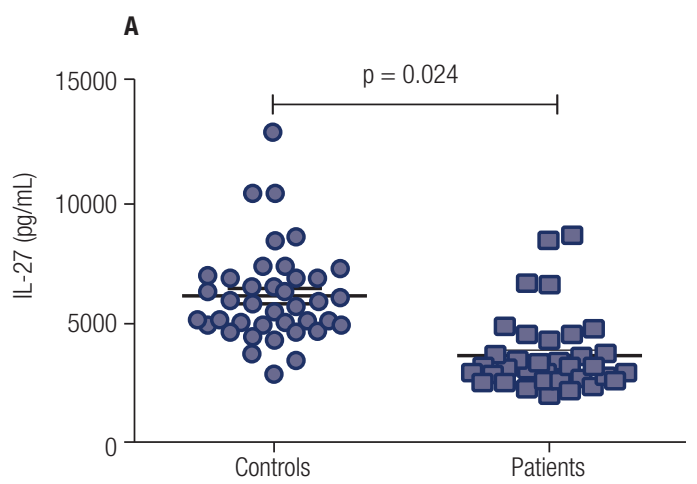

\section{Relationship between IL-27, IL-35, anti-thyroid antibodies, TRAb and the grade of patients}

The relationship between the levels of these two cytokines and the concentration of anti-TPO, anti-Tg antibodies and TRAb levels was examined using the Pearson correlation test. In patients, age did not have any correlations with the levels of IL-27 and IL-35 and TRAb, anti-TPO, and anti-Tg antibodies (Table 2).

There was no significant correlation between any of the measured IL-35, IL-27, TRAb, anti-Tg and anti-TPO factors in the subjects and the disease grading parameter performed by the endocrinologist (Figure 3 and Table 3 ).

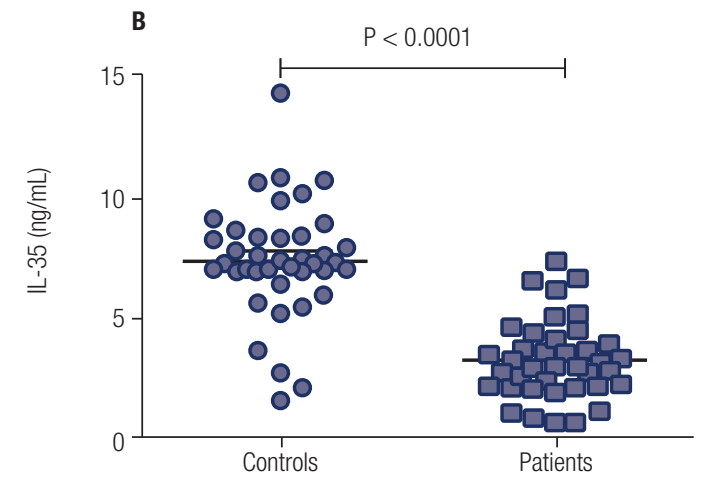

Figure 2. Comparison of the serum levels of IL-27 ( $p=0.024)$ and IL-35 $(p<0.0001)$ in GD patients versus the controls. Cytokine levels in GD patients $(n=40)$ and control group $(n=40)$ were measured by ELISA. Compared to the normal group, the IL-27 and IL-35 were decreased in GD patients.

Table 2. Correlation between IL-27 and IL-35 cytokines and anti-thyroid antibodies and age in patients

\begin{tabular}{lcccc}
\hline & AGE & Anti-TPO & Anti-Tg & trab \\
\hline IL-27 & -0.232 & 0.084 & 0.178 & -0.04 \\
& 0.667 & 0.608 & 0.297 & 0.806 \\
IL-35 & 0.150 & -0.047 & -0.120 & 0.165 \\
& 0.345 & 0.772 & 0.456 & 0.308 \\
\hline
\end{tabular}
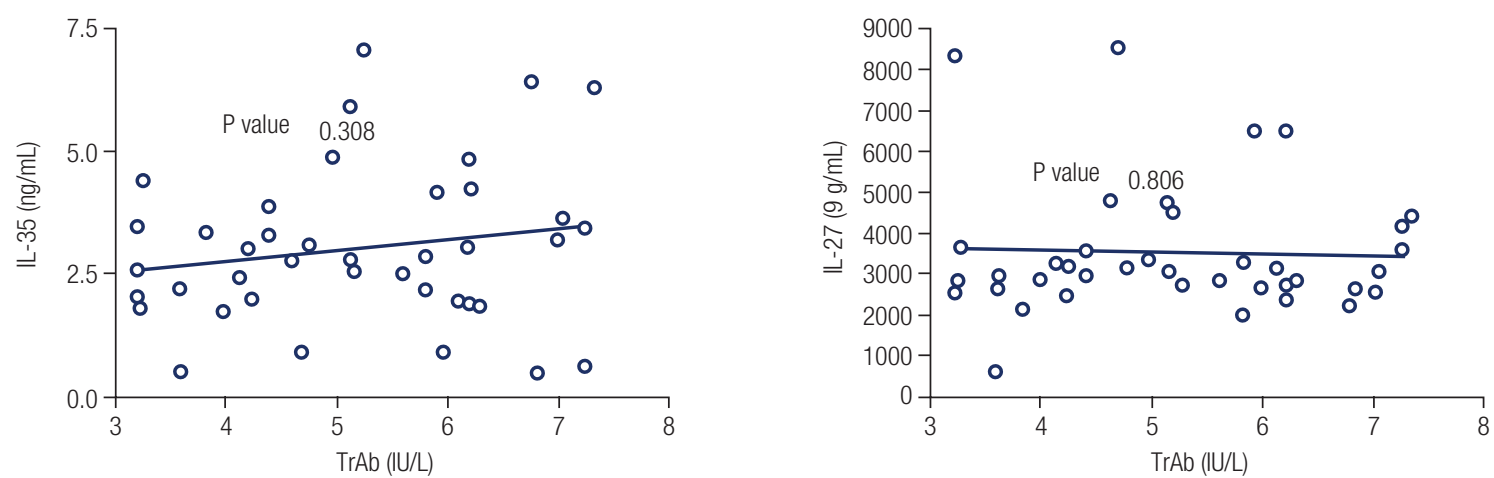

Figure 3. Correlation between IL-27 and IL-35 cytokine levels and level of TRAb in GD patients. 
Table 3. Correlation between grade and IL-27, IL-35 cytokines, anti-thyroid antibodies and age in patients

\begin{tabular}{cccccccc}
\hline & Anti TPO & Anti TG & age & IL35 & IL27 & TRAb \\
\hline \multirow{2}{*}{ Grade } & Pearson Correlation & -0.095 & 0.081 & -0.263 & -0.163 & 0.061 & 0.188 \\
& Sig. (2-tailed) & 0.631 & 0.681 & 0.175 & 0.416 & 0.788 & 0.337 \\
& $N$ & 28 & 28 & 28 & 27 & 22 & 28 \\
\hline
\end{tabular}

No significant correlation was found between IL-27 and IL-35 serum levels and Graves orbitopathy ( $\mathrm{p}=0.74$ and $\mathrm{p}=0.2$, respectively).

\section{DISCUSSION}

GD is a TH2 predominant autoimmune illness defined by the presence of anti-TSH receptor antibody which motivates thyroid hormone secretion (17). The appearance of other antibodies against thyroid antigens such as anti-Tg and anti-TPO antibodies was reported in patients with hyperthyroidism (18). As it was observed in the present study, the serum levels of anti-Tg and anti-TPO antibodies in GD patients was significantly higher than those of control subjects, which is in line with previous reports (19). The generation of these antibodies can be attributed to the increased expression of the specific antigens and loss of tolerance in the $\operatorname{organ}(20)$.

Cytokines take part in the induction and effector steps of all inflammatory and immune responses, playing a critical role in the progression of autoimmune diseases. Excess, reduced, or improper cytokine responses significantly contribute to the formation of autoimmune inflammation (8). In vitro experiment analysis of intra-thyroidal lymphocytes including TSHreceptor specific $\mathrm{T}$ cells has revealed the predominance of the TH2 response in GD. In fact, following the initiation of TH2-related responses, the inflammatory process continues through Thl cells. In this way, the cytokines such as IL-1a, IL- 6 and TNF- $\alpha$ are generated by thyroid follicular cells as well as inflammatory cells. Moreover, increased gene expression of IL-1 $\alpha$, IL-I3, IL-6, TNF- $\alpha$ and IFN- $\gamma$ was also shown in GD (5). On the other hand, IL- $1 \beta$ was able to induce the production of hyaluronan by thyroid epithelial cells and thyroid fibroblasts, a process possibly contributing to the progression of goiter in GD (21). Cytokines of IL-12 family played important roles in the immune system and inflammation (22). IL-27 and IL-35 were relatively new members of the IL-12 family (23), and in the present study, the serum levels of these two cytokines in GD patients were reduced in comparison to normal individuals. IL-27 was shown to have two distinct inflammatory and anti-inflammatory functions (11). To our knowledge, no study has measured IL-27 in GD. Regarding other autoimmune diseases, IL-27 reduction has been reported in multiple sclerosis (MS) patients (24). After the interferon therapy of these patients with IL-27, increased serum levels of IL-27 were observed, and researchers predicted that with the administration of this cytokine, the recovery of MS disease would increase (24). Although increased levels of this cytokine were reported in patients with rheumatoid arthritis compared to healthy subjects (25), the role of IL-27 is complex and in addition to antiinflammatory activity, its pro-inflammatory function should be considered (26). Among IL-27 proinflammatory functions, inhibition of Th17 cells and antagonistic effect on IL-6 activity were reported to be noteworthy $(27,28)$. The dual function of IL-27 may be explained by the fact that this cytokine can be released from various cells such as antigen presenting cells under various conditions depending on the type of disease, cytokine network and the dominant cytokine profile. In this regard, IL-27 was proposed as a promising antiviral and anti inflammatory agent with apparent low toxicity risk as observed in animal and in vitro models (29).

In the present study, a significant reduction was observed in the IL-27 belonging to the sera of patients with GD in comparison to the healthy control group $(\mathrm{P}$ value $=0.024)$. The mean serum level of IL-27 in the patient group was approximately half of the control group. This result casts doubt on the inflammatory role of this cytokine in the development of GD while further confirming the anti-inflammatory properties of this cytokine as evidenced in previous studies on some autoimmune mice models such as uveoretinitis, multiple sclerosis and rheumatoid arthritis (30).

The IL-35 is secreted by activated antigen presenting cells including $\mathrm{B}$ cells, monocytes, macrophages and dendritic cells. This cytokine was initially introduced to be generated by Treg cells and play an essential role in the inhibitory function of these cells (31). Recently, the 
immunomodulatory role of IL-35 has been identified in certain inflammatory conditions (32). The main inhibitory effects of IL-35 were on THI and TH17 cells (31). Administration of IL-35 to mice with type 1 diabetes inhibited the disease (33). Moreover, the therapeutic effects of IL-35 on collagen-induced arthritis in the RA model were documented (34). In the only study on GD, the levels of IL- 35 and TGF- $\beta$ cytokine levels were reduced while IL-17A, IL-23, IL-6 serum levels were increased in Chinese GD patients compared with healthy controls (35), which is consistent with the present study . No correlations were observed between IL-35 and autoantibody levels in the patients of the current research. These results may suggest that this inhibitory cytokine is a therapeutic agent in GD. It is likely that the administration of IL-35 leads to the suppression of related inflammatory process in GD. This hypothesis should be verified in future studies on mice models of autoimmune thyroiditis.

\section{CONCLUSION}

The reduction in the serum levels of IL-27 and IL-35 in GD patients compared to normal subjects suggests the possible anti-inflammatory role of these cytokines in GD, hence their use as therapeutic candidates for the treatment of GD patients in future.

Disclosure: no potential conflict of interest relevant to this article was reported.

\section{REFERENCES}

1. Rapoport B, McLachlan SM. Graves' Disease: Pathogenesis and Treatment. Berlin: Springer Science \& Business Media; 2000.

2. Karnath BM, Hussain N. Signs and symptoms of thyroid dysfunction. Hospital Physician. 2006:43-8.

3. Brent GA. Graves' disease. N Engl J Med. 2008;358(24):2594-605.

4. Alnaqdy A, Al-Maskari M. Levels of cytokines and thyroid autoantibodies in Omani patients with Graves' disease. Br J Biomed Sci. 2007;64(4):164-7.

5. Weetman A, Ajjan RA, Watson P. Cytokines and Graves' disease. Best Pract Res Clin Endocrinol Metab. 1997;11(3):481-97.

6. Shen J, Li Z, Li W, Ge Y, Xie M, Lv M, et al. Th1, Th2, and Th17 cytokine involvement in thyroid associated ophthalmopathy. Dis Markers. 2015;2015:609593.

7. Gianoukakis AG, Khadavi N, Smith TJ. Cytokines, Graves' disease, and thyroid-associated ophthalmopathy. Thyroid. 2008;18(9):953-8.

8. Moudgil KD, Choubey D. Cytokines in autoimmunity: role in induction, regulation, and treatment. J Interferon Cytokine Res. 2011;31(10):695-703.

9. Yoshida H, Miyazaki Y. Regulation of immune responses by interleukin-27. Immunol Rev. 2008;226:234-47.

10. Hall AOH, Silver JS, Hunter CA. The immunobiology of IL-27 Adv Immunol. 2012;115:1-44.
11. Schneider R, Yaneva T, Beauseigle D, El-Khoury L, Arbour N. IL-27 increases the proliferation and effector functions of human naive CD8+ T lymphocytes and promotes their development into Tc1 cells. Eur J Immunol. 2011;41(1):47-59.

12. Owaki T, Asakawa M, Fukai F, Mizuguchi J, Yoshimoto T. IL-27 induces Th1 differentiation via p38 MAPK/T-bet-and intercellular adhesion molecule-1/LFA-1/ERK1/2-dependent pathways. J Immunol. 2006;177(11):7579-87.

13. Villarino AV, Huang $E$, Hunter CA. Understanding the pro-and antiinflammatory properties of IL-27. J Immunol. 2004;173(2):715-20.

14. Yang XF, Li X, Mai J, Virtue A, Yin Y, Sha X, et al. IL-35 Is a Novel Responsive Anti-Inflammatory Cytokine-A New System of Categorizing Anti-Inflammatory Cytokines. Am Soc Hematol. 2012.

15. Li X, Mai J, Virtue A, Yin Y, Gong R, Sha X, et al. IL-35 is a novel responsive anti-inflammatory cytokine-a new system of categorizing anti-inflammatory cytokines. PloS One. 2012;7(3):e33628.

16. Choi J, Leung PS, Bowlus C, Gershwin ME. IL-35 and autoimmunity: a comprehensive perspective. Clin Rev Allergy Immunol. 2015;49(3):327-32.

17. Jia H, Zhang Z, Gu X, Guo T, Cui B, Ning G, et al. Association between interleukin 21 and Graves' disease. Genet Mol Res. 2011;10(4):3338-46.

18. Fröhlich E, Wahl RJ. Thyroid autoimmunity: role of anti-thyroid antibodies in thyroid and extra-thyroidal diseases. Front Immunol. 2017;8:521.

19. Vlainich AR, Romaldini JH, Pedro AB, Farah CS, Sinisgalli Jr CA. Ultrasonography compared to magnetic resonance imaging in thyroid-associated Graves' ophthalmopathy. Arq Bras Endocrinol Metab. 2011;55(3):184-8.

20. Alpdogan $\mathrm{O}$, van den Brink MR, editors. Immune tolerance and transplantation. Semin Oncol. 2012;39(6):629-42.

21. Gianoukakis $A G$, Jennings $T A$, King $C S$, Sheehan $C E$, Hoa $N$, Heldin $P$, et al. Hyaluronan accumulation in thyroid tissue: evidence for contributions from epithelial cells and fibroblasts. Endocrinology. 2007;148(1):54-62.

22. Gee K, Guzzo C, Mat C, Nor F, Ma W, Kumar AJI, et al. The IL-12 family of cytokines in infection, inflammation and autoimmune disorders. Inflamm Allergy Drug Targets. 2009;8(1):40-52.

23. Vignali DA, Kuchroo VK. IL-12 family cytokines: immunological playmakers. Nat Immunol. 2012;13(8):722-8.

24. Hunter C, Kastelein R. Fifteen years of interleukin-27-discovery, advances and translation. Immunity. 2012;37(6):960-9.

25. Lai X, Wang H, Cao J, LiY, Dai Y, Xiang Y, et al. Circulating IL-27 is elevated in rheumatoid arthritis patients. Molecules. 2016;21(11). pii: E1565.

26. Larousserie F, Charlot P, Bardel E, Froger J, Kastelein RA, Devergne O. Differential effects of IL-27 on human B cell subsets. J Immunol. 2006;176(10):5890-7.

27. Batten M, Li J, Yi S, Kljavin NM, Danilenko DM, Lucas S, et al. Interleukin 27 limits autoimmune encephalomyelitis by suppressing the development of interleukin 17-producing T cells. Nat Immunol. 2006;7(9):929-36.

28. Kamiya S, OwakiT, Morishima N, Fukai F, Mizuguchi J, Yoshimoto T. An indispensable role for STAT1 in IL-27-induced T-bet expression but not proliferation of naive CD4+T cells. J Immunol. 2004;173(6):3871-7.

29. Jankowski M, Kopiński P, Goc A. Interleukin-27: biological properties and clinical application. Arch Immunol Ther Exp (Warsz). 2010;58(6):417-25.

30. Stumhofer JS, Hunter CA. Advances in understanding the antiinflammatory properties of IL-27. Immunol Lett. 2008;117(2):123-30.

31. Collison LW, Chaturvedi V, Henderson AL, Giacomin PR, Guy C, Bankoti J, et al. IL-35-mediated induction of a potent regulatory T cell population. Nat Immunol. 2010;11(12):1093-101. 
32. Huang A, Cheng L, He M, Nie J, Wang J, Jiang K. Interleukin-35 on $B$ cell and T cell induction and regulation. J Inflamm (Lond). 2017;14:16.

33. Singh $K$, Kadesjö E, Lindroos J, Hjort M, Lundberg M, Espes D, et al. Interleukin-35 administration counteracts established murine type 1 diabetes - possible involvement of regulatory T cells. Sci Rep. 2015;5:12633.
34. NiedbalaW, Wei XQ, Cai B, Hueber AJ, Leung BP, Mclnnes IB, et al. IL-35 is a novel cytokine with therapeutic effects against collageninduced arthritis through the expansion of regulatory $T$ cells and suppression of Th17 cells. Eur J Immunol. 2007;37(11):3021-9.

35. Lv M, Shen J, Li Z, Zhao D, Chen Z, Wan H, et al. Role ofTreg/Th17 cells and related cytokines in Graves' ophthalmopathy. Nan Fang Yi Ke Da Xue Bao. 2014;34(12):1809-13. 